\title{
Current status of screening, diagnosis, and treatment of neonatal congenital heart disease in China
}

\author{
Xiao-Jing Ma ${ }^{1,2,3} \cdot$ Guo-Ying Huang ${ }^{1,2,3}$
}

Received: 30 June 2018 / Accepted: 6 July 2018 / Published online: 31 July 2018

(c) Children's Hospital, Zhejiang University School of Medicine 2018

Congenital heart disease (CHD) has become the most common birth defect in China. According to the "Report on the Prevention and Control of Birth Defects in China (2012)" [1], in 2011, the prevalence of CHD was 4.095 of every 1000 live births, which accounted for the first place in all birth defects in recent years. Because of the large population base in China, the number of new cases of CHD per year is huge. It is estimated that more than 130 thousand patients with CHD will be added per year in China. According to another study reported in 2014, CHD was present in 8.94 of every 1000 live births in China, among which, the incidence of critical CHD (CCHD), causing death or requiring invasive intervention during the first month of life, was $2.91 \%$ [ [2].

More seriously, $\mathrm{CHD}$ has become the main cause of death in children under 5 years in China. Without timely diagnosis and treatment, approximate $1 / 3$ of children with CHD would die within the first year after birth because of severe anoxia, heart failure, pneumonia, or other serious complications. The life quality of those survival patients would also be severely affected. CHD reduces the health level and quality of the population and the burden of disease caused by treatment; disability or death is tremendous. Based on the reported data in 2003, the total economic burden of new CHD cases in China was more than 12.6 billion Yuan per year [1]. In addition, the CHD and CHD-related complications would bring huge medical burden, economic burden, and mental burden to their family and the society.

At present, the great progress in the field of the diagnosis and treatment of CHD has been achieved. Noninvasive

Guo-Ying Huang

gyhuang@shmu.edu.cn

1 Heart Center, Children's Hospital of Fudan University, 399 Wanyuan Road, Shanghai 201102, China

2 National Children's Medical Center, 399 Wanyuan Road, Shanghai 201102, China

3 Shanghai Key Lab of Birth Defects, 399 Wanyuan Road, Shanghai 201102, China imaging diagnostic techniques, including echocardiography, magnetic resonance imaging, and multi-slice spiral CT, continue to develop, which makes the diagnosis of CHD more accurate. Meanwhile, with the continuous development of cardiopulmonary bypass surgery, catheter intervention, perioperative monitoring, and life support technologies, the survival rate of surgically repaired CHD in some pediatric heart centers, especially in Shanghai, Beijing, Guangzhou, and other developed regions, have reached more than $97 \%$, which is close to the international advanced level. As a minimally invasive technique, interventional therapy for CHD has developed rapidly in China. In 2017, the number of interventional therapy for CHD in the Mainland of China has reached 32,126 cases, with the success rate between [3]. The prognosis of most patients with severe CHD is favourable if they are timely diagnosed and treated appropriately.

However, the contradiction between the increasingly mature technology of diagnosis and treatment and the inability of early detection is the biggest current dilemma in China. It will be life-threatening if the diagnosis of CHD is missed or delayed. Before CCHD added to the US Recommended Uniform Screening Panel for newborns, a considerable portion of CCHD was not detected during the birth hospitalization. Late CCHD detection was significantly associated with $52 \%$ more admissions, $18 \%$ more hospitalized days, and 35\% higher inpatient costs during infancy [4]. Our single center study found that the missed diagnosis rate of severe $\mathrm{CHD}$ before being discharged from birth hospital was $71 \%$ [5]. Therefore, it is very necessary to screen for CHD in the early neonatal period.

Fortunately, the reliable method of neonatal CCHD screening has already been proposed. Based on the evidence provided by several clinical studies, pulse oximetry (POX) has been recommended as a strategy for CCHD screening in neonates by the American Academy of Pediatrics (AAP) [6]. Screening of CCHD in the neonatal period has become a consensus. Thus, more and more countries have introduced this screening as a routine practice. In the United States, screening for CCHD using POX was added 
to the Recommended Uniform Screening Panel in 2011 [7]. Since then, CCHD screening has become nearly universal in the United States. The United Kingdom National Screening Committee was currently investigating the potential feasibility of routine POX screening in their country [8]. In addition, Canadian Cardiovascular Society and Canadian Pediatric Cardiology Association recently recommended that POX screening should be routinely performed in all newborns to enhance the detection of CCHD in Canada [9]. Other countries, such as Switzerland, Poland, Abu Dhabi, and Nordic European countries, have already or are planning to implement CCHD screening program.

However, POX screening finds only those right-to-left shunt cardiac lesions and the detection rate is about $80 \%$. It is not able to detect those critical and serious lesions without hypoxemia, such as left heart obstructive defects and large septal defects. These defects can cause death or require invasive intervention during infancy and should be regarded as the main targets of neonatal screening as well. In two large-sample, prospective, multi-center studies, we found that POX plus cardiac auscultation (double-index method) is able to detect over $92 \%$ of major CHD (including critical and serious lesions) with reasonable false-positive rate $[2,10]$. Based on these work, in April 2016, Shanghai Municipal Commission of Health and Family Planning recommended CHD screening should be carried out in all newborns [11], which has started since June 1, 2016. Up to March 31, 2018, the overall screening rate has reached $99.36 \%$ (unpublished data). Meanwhile, the referral system of CHD in Shanghai has been established and it can serve as a reference for other regions in China.

Although the hierarchical treatment and referral system has not yet been established throughout the country [12], the importance and feasibility of neonatal CHD screening have been demonstrated in China [13]. The integrated system consisting of screening, referral, and information transmission will make it possible for early detection, timely diagnosis, and appropriate management of CHD in the country.

It should be mentioned that the screening strategy for CHD should also keep pace with the improvements and developments of prenatal diagnosis techniques, such as fetal echocardiography, genetic testing, and metabolic markers detection. It is undoubtedly more effective in improving the prognosis of the patients if it is possible to discover and diagnose CHD, especially the critical lesions, before birth, together with the fetal interventions and perinatal managements.

Author contributions GYH and XJM drafted the initial manuscript. Both authors reviewed, revised, and approved the final manuscript.
Funding This study was supported by National Key Research and Development Program (2016YFC1000500), Shanghai Clinical Medical Center for pediatric diseases (2017ZZ01008), and Shanghai Public Health Three-Year Action Plan sponsored by Shanghai Municipal Government (Nos. 2011-55 and 2015-82).

\section{Compliance with ethical standards}

Ethical approval Not needed.

Conflict of interest The authors have no financial relationships relevant to this article to disclose.

\section{References}

1. National Health Commission of the People's Republic of China. Report on the prevention and control of birth defects in China. 2012. http://www.gov.cn/gzdt/att/att/site1/20120912/1c6f6506c7 f811bacf9301.pdf. Accessed 27 June 2018 (in Chinese).

2. Zhao QM, Ma XJ, Ge XL, Liu F, Yan WL, Wu L, et al. Pulse oximetry with clinical assessment to screen for congenital heart disease in neonates in China: a prospective study. Lancet. 2014;384:747-54.

3. Data on interventional treatment for congenital heart disease in mainland China. 2017. http://meeting.dxy.cn/article/557035. Accessed 27 June 2018 (in Chinese).

4. Peterson C, Dawson A, Grosse SD, Riehle-Colarusso T, Olney RS, Tanner JP, et al. Hospitalizations, costs, and mortality among infants with critical congenital heart disease: how important is timely detection? Birth Defects Res A Clin Mol Teratol. 2013;97:664-72.

5. Zhao QM, Liu F, Wu L, Ye M, Jia B, Ma XJ, et al. Assessment of undiagnosed critical congenital heart disease before discharge from the maternity hospital. Zhonghua Er Ke Za Zhi. 2017;55:260-6.

6. Kemper AR, Mahle WT, Martin GR, Cooley WC, Kumar P, Morrow WR, et al. Strategies for implementing screening for critical congenital heart disease. Pediatrics. 2011;128:e1259-67.

7. Recommended Uniform Screening Panel. https://www.hrsa. gov/advisory-committees/heritable-disorders/rusp/index.html. Accessed 24 July 2018.

8. Ismail AQT, Cawsey M, Ewer AK. Newborn pulse oximetry screening in practice. Arch Dis Child Educ Pract Ed. 2017;102:155-61.

9. Wong KK, Fournier A, Fruitman DS, Graves L, Human DG, Narvey M, et al. Canadian Cardiovascular Society/Canadian Pediatric Cardiology Association position statement on pulse oximetry screening in newborns to enhance detection of critical congenital heart disease. Can J Cardiol. 2017;33:199-208.

10. Hu XJ, Ma XJ, Zhao QM, Yan WL, Ge XL, Jia B, et al. Pulse oximetry and auscultation for congenital heart disease detection. Pediatrics. 2017;140:e20171154.

11. Notification on screening neonatal congenital heart disease in the city. http://www.wsjsw.gov.cn/wsj/n429/n432/n1487/n1511/u1ai1 37540.html. Accessed 24 July 2018 (in Chinese).

12. Liu Y, Yang LL, Xu SY, Zhao ZY. Pediatrics in China: challenges and prospects. World J Pediatr. 2018;14:1-3.

13. Maternity and Child Health Department, National Health Commission of China. Notice on the application for screening of neonatal congenital heart disease; 2018. 